\title{
Allaitement maternel: trois communiqués
}

A l'occasion de l'Année internationale de l'enfant 1979 et de la parution de ce numéro de Médecine sociale et préventive, il nous paraît indiqué de reprendre trois communiqués publiés récemment dans des périodiques du domaine de la santé internationale:

\section{Réglementation sur les biberons en Papouasie-Nouvelle Guinée}

La Papouasie-Nouvelle-Guinée parait être le premier pays à avoir adopté des textes législatifs limitant la vente des biberons et des tétines, qui ne peuvent plus être obtenus que sur ordonnance d'un médecin, d'un assistant médical ou d'une infirmière. Le but recherché est d'encourager l'allaitement au sein. En outre, la politique adoptée par le gouvernement de ne pas accepter de dons de produits alimentaires en provenance d'outre-mer, sauf en cas d'urgence, favorisera la production d'aliments de sevrage locaux.

La Papouasie-Nouvelle-Guinée a lancé un programme de nutrition et met rapidement sur pied une politique nationale d'alimentation et de nutrition. Pour plus de renseignements sur ces questions, s'adresser au $\mathrm{Dr}$ N. V. K. Nair (Bureau régional OMS du Pacifique occidental, P.O. Box 2932, 12115 Manille, Philippines).

(Tiré de la Chronique OMS 32, p. 512, décembre 1978)

\section{Mexique - Rien ne vaut l'allaitement maternel}

La campagne en faveur de l'allaitement maternel, lancée en juillet dernier par l'Institut mexicain de la sécurité sociale, a pour objet d'encourager les mères à revenir à une méthode d'alimentation naturelle. La publicité faite à la télévision et dans la presse souligne la valeur nutritionnelle, immunologique et émotionnelle que l'allaitement au sein apporte à l'enfant, ainsi que ses avantages pour la mère.

La campagne est le résultat d'une enquête menée par le Département de gynécologie et d'obstétrique de l'Institut, à Mexico, ainsi que la Division de biologie reproductive en collaboration avec le Sous-secrétariat pour l'amélioration de l'environnement. L'étude montra que, si $80 \%$ des Mexicaines sont capables d'allaiter leur enfant, $11 \%$ seulement dans les villes et $20 \%$ dans les zones rurales choisissent cette méthode pendant une période moyenne de quatre mois.

Les médecins de l'Institut considèrent que les femmes devraient être encouragées à allaiter leur enfant à la maternité déjà. Malheureusement, elles reçoivent souvent des boîtes de lait en poudre de fabricants.

(Tiré de Peuples, International Planned Parenthood Federation, Londres, Vol. 6, No 1, p. 35, 1979)

L'allaitement maternel est une priorité aussi dans les pays industrialisés

Courrier des lecteurs de "Contact»

De Saint-John's, Terre-Neuve, Canada:

«La Société canadienne de pédiatrie a décidé que l'an prochain - Année internationale de l'enfant - tous les pédiatres canadiens mettraient l'accent sur le développement de l'allaitement au sein. Notre objectif est d'accroître de $100 \%$ l'allaitement au sein au-delà de deux mois dans tout le Canada avant la fin de 1979. Pour atteindre cet objectif, il faudra que chaque pédiatre du pays entreprenne personnellement d'évaluer la situation dans sa région. Nous nous attendons à un succès, et nous espérons que d'autres pays pourront se fixer le même but pour l'Année internationale de l'enfant. C'est là certainement une priorité urgente.»

Dr Elisabeth S. Hillman

(Tiré de Contact, No 38, p. 14, novembre 1978, Commission médicale chrétienne, Conseil cecuménique des Eglises, 1211 Genève 20)

Enfin, relevons que Famille et Développement (voir article de P. Pradervand dans ce cahier) présente dans son numéro 17 de janvier 1979 un dossier sur l'alimentation au sein, sous le titre «Cet inimitable lait maternel». 